\title{
BMJ Open Protocol for ACCESS: a qualitative study exploring barriers and facilitators to accessing the emergency contraceptive pill from community pharmacies in Australia
}

Safeera Yasmeen Hussainy, ${ }^{1}$ Ayesha Ghosh, ${ }^{1}$ Angela Taft, ${ }^{2}$ Danielle Mazza, ${ }^{3}$ Kirsten Isla Black, ${ }^{4}$ Rhonda Clifford, ${ }^{5}$ Sajni Gudka, ${ }^{5}$ Kevin Peter Mc Namara, ${ }^{1,6}$ Kath Ryan, ${ }^{7}$ John Keith Jackson ${ }^{1}$

To cite: Hussainy SY, Ghosh A, Taft A, et al. Protocol for ACCESS: a qualitative study exploring barriers and facilitators to accessing the emergency contraceptive pill from community pharmacies in Australia. BMJ Open 2015;5 e010009. doi:10.1136/ bmjopen-2015-010009

- Prepublication history for this paper is available online. To view these files please visit the journal online (http://dx.doi.org/10.1136/ bmjopen-2015-010009).

Received 16 September 2015 Revised 8 November 2015 Accepted 12 November 2015

CrossMark

For numbered affiliations see end of article.

\section{Correspondence to}

Dr Safeera Yasmeen

Hussainy; safeera.hussainy@ monash.edu

\section{ABSTRACT}

Introduction: The rate of unplanned pregnancy in Australia remains high, which has contributed to Australia having one of the highest abortion rates of developed countries with an estimated 1 in 5 women having an abortion. The emergency contraceptive pill (ECP) offers a safe way of preventing unintended pregnancy after unprotected sex has occurred. While the ECP has been available over-the-counter in Australian pharmacies for over a decade, its use has not significantly increased. This paper presents a protocol for a qualitative study that aims to identify the barriers and facilitators to accessing the ECP from community pharmacies in Australia.

Methods and analysis: Data will be collected through one-on-one interviews that are semistructured and in-depth. Partnerships have been established with 2 pharmacy groups and 2 women's health organisations to aid with the recruitment of women and pharmacists for data collection purposes. Interview questions explore domains from the Theoretical Domains Framework in order to assess the factors aiding and/or hindering access to ECP from community pharmacies. Data collected will be analysed using deductive content analysis. The expected benefits of this study are that it will help develop evidencebased workforce interventions to strengthen the capacity and performance of community pharmacists as key ECP providers.

Ethics and dissemination: The findings will be disseminated to the research team and study partners, who will brainstorm ideas for interventions that would address barriers and facilitators to access identified from the interviews. Dissemination will also occur through presentations and peerreviewed publications and the study participants will receive an executive summary of the findings. The study has been evaluated and approved by the Monash Human Research Ethics Committee.

\section{Strengths and limitations of this study}

- Contribution of new knowledge to the limited literature on barriers to emergency contraceptive pill access in the Australian context that will help inform the development and implementation of interventions in community pharmacy to enhance access.

- Use of the Theoretical Domains Framework that includes constructs from 33 behaviour change theories, to conduct and analyse the interviews and ultimately inform the interventions.

- Recruitment challenge is an anticipated problem due to the sensitive nature of the research topic and the fear of disclosure or privacy concerns, potentially delaying the study timeline.

\section{INTRODUCTION}

The rate of unplanned pregnancy in Australia remains high and nearly half of all Australian women of reproductive age have experienced an unplanned pregnancy. ${ }^{1}$ Australia is reported to have one of the highest abortion rates of developed countries with an estimated one in five women having an abortion. ${ }^{2}$ The emergency contraceptive pill (ECP) offers a way of preventing unintended pregnancy after unprotected sex has occurred. It is important to note that many of the previous randomised controlled trials $^{3-6}$ demonstrating no association between ECP access or its advanced provision and unintended pregnancy or abortion rates have mostly been conducted in postpartum or family management clinics or hospitals, and thus targeted women who were already accessing specialised forms of care. However, one trial has demonstrated that increasing access to the ECP can reduce unintended 
pregnancy rates during breast feeding, ${ }^{7}$ highlighting the importance of the culture, setting and context of such trials. Women in general health settings such as community pharmacy may have different attitudes, needs and health-seeking behaviours, and the care they receive may not be as systematic or evidence-based. Further, relationships between women and community pharmacists, and women and general practitioners (GPs), may also be different, with pharmacy being challenged by the consumer's power in the commercial transaction and perceived expertise in the management of minor illness. It is therefore critical to study these populations in greater depth than what has already been investigated, ${ }^{4} 8$ that is, in several countries and different cultural contexts, to determine an effect of enhanced ECP access on unintended pregnancy rates.

While the ECP is safe and has no medical contraindications, there are significant barriers to pharmacy access in Australia and overseas. ${ }^{9}$ Barriers such as suboptimal acceptance by healthcare providers and the public, and multiple financial and healthcare system barriers to use ${ }^{10}$ are preventing the ECP's potential for reducing unintended pregnancies and abortion rates to be realised. The only ECP in Australia, containing levonorgestrel, has been available since 2004 through community pharmacies as a 'Pharmacist Only Medicine' without a prescription. In Australia, Pharmacist Only Medicines must be stored in a part of the pharmacy not accessible to the public and supplied only for a therapeutic need after the pharmacist has personally taken reasonable steps to ensure that such a need exists. The Pharmaceutical Society of Australia (PSA) released the first protocol to guide pharmacists' supply of the ECP in $2003^{11}$ and released an updated version in $2006 .{ }^{12}$ In 2011, the PSA revised the 2006 protocol and released another updated practice guideline for levonorgestrel provision that contains the latest scientific evidence regarding its use. ${ }^{13}$ The revisions address several factors including the time frame that levonorgestrel can be used within, which was changed to allow use up to $96 \mathrm{~h}$ after intercourse, compared with the product information that indicated use within $72 \mathrm{~h}$. The revision also established that advance supply does not negatively impact on sexual and reproductive health. Lastly, there was an acknowledgement that there is limited data regarding the use of the ECP in females aged 1416 years, and the pharmacist needs to refer to a GP where advisable; however, the guideline highlighted that there was no reason for ECP use to be restricted on the basis of age.

Pharmacists' practices in Australia are variable, commonly not meeting evidence-based recommendations in the PSA guideline and resulting in women being unnecessarily declined ECP supply. ${ }^{14}{ }^{15}$ Women's experiences of obtaining the ECP from pharmacies are both positive and negative. ${ }^{9}$ Some positive experiences reported by women include faster and more direct access, convenient location, and feeling of more control over their reproductive health, while some negative experiences reported include lack of privacy, judgemental or indifferent pharmacist attitude, cost of ECP and so on. Compounding this is the unexplained paradox between unplanned pregnancy rates and ECP availability. ${ }^{16}$

Access to emergency contraception, especially the ECP, is essential as it helps prevent unwanted pregnancies-an important public health goal. If the ECP is refused by a pharmacist or GP, women are placed at risk of having an unwanted pregnancy that may result in an abortion or be carried to term with long-term implications for the woman and her partner. This study seeks to understand the underpinning reasons for refusal of supply that make access to this medicine unnecessarily complex.

The ECP was made available over-the-counter (OTC) in Australia with the view that it would allow women to access the ECP more quickly than from a GP, and therefore lead to a decrease in the unplanned pregnancy rate in Australia. A focus group study suggested that Australian women aged 16-30 years were in favour of pharmacy availability of the ECP, as faster and more direct access is afforded, particularly on Sundays and for women living in rural and remote locations. ${ }^{17}$ However, a decade later, despite pharmacy availability of the ECP, it seems that Australian women's use of the ECP has neither significantly increased nor has the rate of unplanned pregnancies significantly decreased. A study conducted in Sydney that surveyed 718 women on ECP use concluded that OTC availability and access to the ECP increased women's awareness but did not significantly increase ECP use among abortion seekers. ${ }^{18}$

A research study examining the attitudes and practices of pharmacists in Australia in relation to their increased role in ECP provision following the policy change to OTC availability found that pharmacists' attitudes and beliefs play a major role in ECP dispensing. ${ }^{15}$ Australian pharmacists had stronger and more conservative views than overseas pharmacists, and $22 \%$ of the pharmacists surveyed felt it was reasonable for a pharmacist's religious faith to influence ECP supply. This seems stark compared with the survey response of pharmacists in Nova Scotia, Canada, where only $1.6 \%$ of pharmacists indicated that they had not provided the ECP due to moral, religious or ethical objections. ${ }^{19}$ In addition, pharmacists' decision to decline ECP provision in Australia is because of incorrect beliefs regarding advance prescription, the responsible use of ECP and its impact on sexual reproductive health. ${ }^{15}$ Pharmacists also noted a number of problems with the number of differing written protocols used to dispense the ECP. In another study examining attitudes of pharmacy assistants in Northern Queensland, 22\% of those interviewed felt it was reasonable for a pharmacists' religious faith to influence ECP supply, while $65 \%$ of pharmacists interviewed identified young age as the most common reason for refusing to dispense ECP. ${ }^{20}$ 
The first random population-based study of Australian women's ECP knowledge, attitudes and use since its availability without a prescription surveyed 632 Australian women aged 16-35 years. ${ }^{9}$ This study found that less than half were aware that ECP was available from pharmacies without a prescription and $57 \%$ did not think they were at risk of getting pregnant. While most women felt the ECP was effective at preventing pregnancy, less than half believed that is was safe or very safe for the health of women. Of the women surveyed, $32 \%$ thought that the ECP was an abortifacient, when in fact, it delays ovulation. In addition, more than half the women reported feeling somewhat or very uncomfortable when asking for the ECP at a pharmacy and less than half thought it was the role of the pharmacist to give women advice about contraception and sexually transmissible infections at the time the ECP is obtained. Although Australian women have a high awareness of the ECP, their knowledge about how and when to use it and where to obtain it is inadequate, thus increasing their risk of becoming pregnant. ${ }^{9}$

A comprehensive barriers analysis to determine pharmacist-related and patient-related barriers to ECP provision has not been done in Australia or overseas, although some light has been indirectly thrown onto this issue in a previous mystery caller study of a sample of pharmacies in Victoria, Australia. ${ }^{14}$ In this study, 515 pharmacists were randomly allocated one of three scenarios when supplying the ECP and these scenarios exemplified the three major areas of change in the revised PSA guideline: outside the licensed $72 \mathrm{~h}$ time frame (scenario 1); by a woman under 16 years (scenario 2); and for future use (scenario 3). These scenarios tested actual performance in situations for which pharmacists' self-reported responses in a previous study were inappropriate. ${ }^{15}$ It was found that $55.4 \%$ of pharmacists tested for scenario 1 declined supply and most referred to the doctor; and $46.1 \%$ and $40 \%$ denied supply for scenarios 2 and 3, respectively. The study concluded that Victorian pharmacists' practices in relation to ECP provision are not always in line with the recommendations in the PSA guideline.

These findings are mirrored by a recent review of workforce interventions that facilitate increased access to ECP in low-income and middle-income countries, revealing that in these countries too, provider knowledge gaps, less than favourable attitudes and practice issues impact access to ECP. The review also highlighted the need to further examine provider performance to inform the development of appropriate workforce interventions. $^{21}$

We therefore recommend that a formal analysis is required to understand how services such as community pharmacy should be reoriented to ensure they meet the sexual and reproductive health needs of women in Australia. Hence, in-depth interview with key stakeholders-women and pharmacists-is the proposed method to undertake the barriers analysis in this
Australian-based study that we have named ACCESS (ACcessing Contraception for Emergency Supply Study). In-depth interviewing will be used to develop an understanding of both individual (attitudinal, knowledgebased, skills-related, risk assessment) and organisational barriers and facilitators. Key informant interviews with pharmacists as well as key informant interviews with women living in Australia will be conducted over a 4-month period. The interview questions have been developed based on the Theoretical Domains Framework (TDF) ${ }^{22}$ and seek information pertaining to women's interactions with pharmacists when obtaining the ECP, attitudes and beliefs of pharmacists and women, as well as characteristics of those interviewed (gender, age, highest education level, country of birth, primary language spoken, place of residence, employment status, type of health insurance, marital status and other relevant characteristics).

The major significance of ACCESS will be the evidence that it will provide to help inform workforce interventions in community pharmacy that will address barriers to ECP access, promote increased adherence with the PSA national guideline and therefore increase supply of, and enhance access to, the ECP by women. A key focus of the study is to evaluate practice against national guideline evidence in order to facilitate ECP supply. The data from this study will be used to develop and pilot evidence-based interventions that will strengthen the capacity of pharmacists to play a more effective role in reducing unwanted pregnancies and the abortion rate in Australia.

\section{METHODS AND ANALYSIS \\ Design \\ Exploratory qualitative study.}

\section{Scope}

The study will be carried out in collaboration with 10 pharmacy sites located in Victoria, Australia. We will work with our partners to include pharmacies from various different locations/regions within Victoria as well as pharmacists from differing religious affiliations and genders. The pharmacist interview will take place at the pharmacy where they work and the women interviewees will be asked to nominate a mutually agreeable place such as the Monash University Parkville campus where the chief investigator is based or over the phone. The study will be carried out over a period of 12 months where after gaining ethics approval, 3 months will be designated for recruitment and 4 months towards conducting the interviews which will be followed by a few months of data analysis.

\section{Sample size}

The sample size of pharmacists we seek to interview is anywhere between 10 and 50 participants. The sample size of women participants we seek to interview is 
Table 1 Interview guide for pharmacists according to Michie's theoretical domains

\begin{tabular}{|c|c|}
\hline Theoretical domains & Interview prompts \\
\hline \multirow[t]{2}{*}{ Knowledge } & Are you aware of the PSA guideline for providing the ECP? \\
\hline & What is your understanding of this guideline? \\
\hline \multirow[t]{2}{*}{ Skills } & Have you had any training to use the PSA guideline? What kind of training? \\
\hline & $\begin{array}{l}\text { What skills are required to supply ECP to someone? Are there any specific areas of } \\
\text { difficulty? }\end{array}$ \\
\hline \multirow{3}{*}{$\begin{array}{l}\text { Social/professional role and } \\
\text { identity }\end{array}$} & Why do you provide the ECP in your pharmacy? \\
\hline & $\begin{array}{l}\text { What are your views about the PSA guideline in general? Do you think it is an appropriate } \\
\text { part of your role to be following this guideline? }\end{array}$ \\
\hline & $\begin{array}{l}\text { Does your ethical position affect your practice with regard to the ECP? How do you } \\
\text { reconcile this with your duty of care? }\end{array}$ \\
\hline \multirow[t]{3}{*}{ Beliefs about capabilities } & $\begin{array}{l}\text { Do you find it difficult to apply the information in the PSA guideline to assess whether } \\
\text { someone should receive the ECP? What problems have you encountered? What would } \\
\text { help you to overcome these problems? }\end{array}$ \\
\hline & Do you think you have the skills to provide the ECP? \\
\hline & $\begin{array}{l}\text { Do you fear that you might miss something when assessing whether someone should } \\
\text { receive the ECP? }\end{array}$ \\
\hline $\begin{array}{l}\text { Memory, attention and decision } \\
\text { processes }\end{array}$ & What thought processes might guide your decision to provide the ECP to someone? \\
\hline \multirow[t]{3}{*}{ Beliefs about consequences } & $\begin{array}{l}\text { In your experience of providing the ECP, have you come across problems in your } \\
\text { population? }\end{array}$ \\
\hline & What do you think about the evidence behind the ECP? \\
\hline & $\begin{array}{l}\text { Are there any advantages or disadvantages in trying to access the ECP via a pharmacy } \\
\text { instead of a health clinic or doctor? }\end{array}$ \\
\hline Behavioural regulation & $\begin{array}{l}\text { Are there any procedures or ways of working that encourage or discourage you to provide } \\
\text { the ECP? }\end{array}$ \\
\hline \multirow[t]{2}{*}{ Social influences } & $\begin{array}{l}\text { To what extent do social influences of peers, managers, etc, facilitate or hinder you in... } \\
\text { Providing the ECP? }\end{array}$ \\
\hline & Applying the PSA guideline? \\
\hline \multirow{4}{*}{$\begin{array}{l}\text { Environmental context and } \\
\text { resources }\end{array}$} & Are there any environmental or resource factors that facilitate or hinder you in... \\
\hline & Providing the ECP? \\
\hline & Applying the PSA guideline? \\
\hline & Does your pharmacy use any checklists or tools when providing the ECP? \\
\hline \multirow[t]{3}{*}{ Nature of the behaviours } & What do pharmacies have to do differently to... \\
\hline & Improve awareness and access of the ECP? \\
\hline & Increase the application of the PSA guideline? \\
\hline
\end{tabular}

anywhere between 20 and 70 participants. The reason for such a wide range in sample size is due to the fact that since this is an exploratory qualitative study, the number of interviews is dependent on whether saturation of themes is reached and is dictated by resources.

\section{Tool}

Participants will be interviewed for between $30 \mathrm{~min}$ and $1 \frac{1}{2} \mathrm{~h}$, and they will be asked open-ended questions focusing on the barriers and facilitators to accessing ECP from community pharmacies. The interview questions were formulated based on Michie's TDF ${ }^{22} 23$ described in detail below.

Pharmacists will be encouraged to talk about internal beliefs and attitudes that may hinder them from freely providing the ECP. The 21 interview questions explore the following 10 behavioural constructs in order to assess pharmacist-related barriers to ECP provision: knowledge; skills; social/professional role and identity; beliefs about capabilities; memory, attention and decision processes; beliefs about consequences; behavioural regulation; social influences; environmental context and resources; and nature of behaviour (table 1).

Women will be encouraged to talk about the social pressures, judgements and prior experiences that may hinder them from accessing the ECP from pharmacies. The 14 interview questions regarding consumer-related barriers to ECP provision explore all the same behavioural constructs mentioned above except for one: memory, attention and decision processes (table 2).

\section{Theoretical Domains Framework}

Behaviour change is a key to increasing the uptake of evidence into healthcare practice and improving health outcomes. A variety of psychological theories have been used to explain healthcare professional behaviours and cognitions across a range of behaviours and settings. However, the large number of theories and overlapping 
Table 2 Interview guide for women according to Michie's theoretical domains

\begin{tabular}{|c|c|}
\hline Theoretical domains & Interview prompts \\
\hline \multirow[t]{4}{*}{ Knowledge } & What is the ECP? \\
\hline & How long after unprotected sex can you take the ECP? \\
\hline & Where would you get the ECP from? \\
\hline & Did you know the ECP is available without a prescription from a pharmacy? \\
\hline \multirow[t]{2}{*}{ Skills } & Have you ever taken the ECP? From where? When? \\
\hline & $\begin{array}{l}\text { (Note: if they got it from pharmacy-then the question is complete. If they got it from } \\
\text { somewhere else, and didn't know about pharmacy access-then ask would they if they } \\
\text { could?) }\end{array}$ \\
\hline Beliefs about capabilities & What can be done to increase someone's capability to access the ECP from a pharmacy? \\
\hline Beliefs about consequences & $\begin{array}{l}\text { Are there any advantages or disadvantages in trying to access the ECP via a pharmacy } \\
\text { instead of a health clinic or doctor? }\end{array}$ \\
\hline \multirow[t]{3}{*}{ Behavioural regulation } & We want women to know that it is easy, convenient and fast to get the ECP from pharmacies. \\
\hline & What factors are important to you if you had to get the ECP from a pharmacy? \\
\hline & $\begin{array}{l}\text { (Prompt: good factors include convenient location, fast service, avoidance of doctor's visit } \\
\text { and so on; bad factors include lack of privacy, fear of judgement and so on) }\end{array}$ \\
\hline \multirow{2}{*}{$\begin{array}{l}\text { Social/professional role and } \\
\text { identity }\end{array}$} & What skills do you think a pharmacist should have when providing the ECP? \\
\hline & Who do you think has the skills to provide the ECP? \\
\hline \multirow{2}{*}{$\begin{array}{l}\text { Environmental context and } \\
\text { resources }\end{array}$} & Is there anything about the pharmacy environment that concerns you? \\
\hline & What information do you think a pharmacist should be able to provide you with? \\
\hline Social influences & $\begin{array}{l}\text { Do you know people who have accessed the ECP from a pharmacy? What problems did they } \\
\text { encounter? Have their experiences facilitated or hindered you in accessing the ECP? }\end{array}$ \\
\hline Nature of the behaviours & What do pharmacies have to do differently to improve awareness of and access to the ECP? \\
\hline
\end{tabular}

constructs presents a challenge for knowing how to select and apply theories when exploring specific behaviours. The TDF, which includes constructs from 33 behaviour change theories, was developed to make theories more accessible for implementation researchers. $^{23}{ }^{24}$ TDF consists of 14 theoretical domains and exemplar questions for each to use in interviews or focus groups to provide a comprehensive theoretical assessment of implementation problems. This framework has been used by research teams across several healthcare systems to explain implementation problems and inform implementation interventions. The TDF has proved useful across a number of healthcare systems and for stronger explanatory and predictive power, and therefore increased usefulness in informing interventions to improve implementation and bring about other behaviour change. ${ }^{23}$

In a brief review to assess the extent of TDF-based research, 133 papers that cite the framework were identified. $^{23}$ Of these, 17 used the TDF as the basis for empirical studies to explore health professionals' behaviour. The identified papers provide evidence of the impact of the TDF on implementation research. Two major strengths of the framework are its theoretical coverage and its capacity to elicit beliefs that could signify key mediators of behaviour change. The TDF has been applied in many implementation studies. ${ }^{22}$ Specifically, qualitative studies have concluded that the TDF was useful for the comprehensive exploration of possible explanations for suboptimal implementation behaviour and for the identification of suitable theories to further investigate those behaviours. ${ }^{25}$ Another study documenting the development and use of the TDF stated that the TDF is arguably the most comprehensive framework for designing implementation interventions as it offers a broad coverage of potential change pathways. ${ }^{26}$ An example of a study utilising TDF to inform the design of its intervention is the Healthy Kids Check (HKC). The authors of this study concluded that TDF was able to classify which barriers needed to be targeted to improve implementation of HKC services. ${ }^{27}$ The study reported here aims to do the same by using the TDF to identify those behavioural constructs that will need to be targeted in order to increase pharmacy performance in ECP provision and ultimately women's access to the ECP.

\section{Recruitment}

The pharmacy organisations involved in this researchAustralian Pharmaceutical Industry (API)/Priceline Pharmacy Group whose main clientele are women and Quality Pharmacy Group (QPG) who is focused on professional service delivery-will nominate five pharmacies each in their group as the sampling frame, that is, total of 10 pharmacies in Victoria.

Every pharmacist working at the nominated pharmacies of both pharmacy groups will be given an information pack by their organisation that will contain information about the study. Pharmacists interested in participating in the study will contact the researchers to enrol in the study.

Women participants will be recruited by API/Priceline Pharmacy Group by advertising the study in an in-store 
leaflet that will be provided to consumers (eg, placed in store bags, placed on counters for consumers to selfselect). QPG will select women participants from their database of approximately 70000 pharmacy consumers. This database contains both demographic and medication-related information, that is, prescription and non-prescription medicines purchased by consumers.

Additional recruitment methods will be employed if recruiting women participants through the two pharmacy groups aforementioned generates a low response. These additional recruitment strategies will be carried out by the Policy and Health Promotion Manager from Women's Health Victoria and the Marketing and Communications Director from Marie Stopes International (partner institutions). Women's Health Victoria will recruit through their networks statewide by sending the recruitment flyer to all the managers and staff in their network of Victorian Women's Health Programs and School Nursing Programs. Marie Stopes International will aid in recruitment by posting the recruitment flyer on the 'Morning After Pill' webpage on the Marie Stopes International website. Lastly, Fernwood fitness clubs, which have exclusively female membership, will be approached to aid in recruitment by displaying the recruitment flyer at selected locations in the gym such as the reception area and women's changing rooms. Fernwood fitness managers will be incentivised with a $\$ 75$ voucher for recruiting at least four women participants.

The research assistant (AG, MPH, female, has experience in conducting and analysing interviews) will contact women who are selected for the study after they have been screened for the inclusion/exclusion criteria, to determine the date, time and interview location that suits them. Similarly, pharmacists selected for the study will also be contacted in order to determine a suitable date and time for the interview. No prior knowledge or characteristics about the research assistant will be shared with the participants, or relationship with her will be established, prior to the interviews.

\section{Inclusion criteria}

In order to participate in the study, pharmacists should be over the age of 18 and English should be their primary language. The women participating in the study should be between the ages 15 and 44 and English should also be their primary language.

\section{Exclusion criteria}

Pharmacists who have never refused supply of the ECP will be excluded from the study. The reason we included this criterion is because the majority of our interview questions aim at eliciting answers to situations where a pharmacist has refused ECP access. In addition, women who have not tried to access the ECP within the past year will be excluded from the study. This is because we want our participants to be able to describe in detail (with minimal recall bias) their prior experience of accessing the ECP.

\section{Compensation}

A $\$ 75$ gift voucher will be given to pharmacist and women interviewees.

\section{Data analysis}

All interviews will be tape-recorded and conducted by the research assistant who does not intend to make field notes during or after. No one else besides the research assistant and interviewee will be present and repeat interviews will not be conducted. Interview data will be transcribed. Participants will receive a copy of their transcript for approval. Data from the interviews will be de-identified, so that no participant names or other identifying features will appear in any form of data reporting. Instead, codes will be used to identify who the comment or quote was made by in the interview. Transcripts will be read and coded by the research assistant, then checked by the chief investigator and one other investigator. The coding process will be deductive with the selected theoretical domains as an organising framework. If the codes validate the TDF domains, they will be used to inform appropriate workforce interventions for a pilot randomised controlled trial in selected API/Priceline and QPG community pharmacies.

NVivo (V.10) will be used to manage data and code these data into TDF domains. Data will be retained in the Centre for Medicine Use and Safety at Monash University, Parkville, for at least 5 years. Hard copies of data will be kept in a locked filing cabinet, in a locked room at the Centre. All electronic data will be stored in password-protected computers. Only the study team will have access to the data.

Possible outcome of the analysis and benefits of the study A study on barriers and facilitators to accessing the ECP from community pharmacy in Australia has never been undertaken before. We hope the data from this study will help develop evidence-based workforce interventions to strengthen the capacity and performance of community pharmacists as key ECP providers.

Possible benefits are that the study will contribute new knowledge to the limited literature on barriers to ECP access in the Australian context and will help inform the development of evidence-based interventions in community pharmacy that will achieve the following: address barriers to ECP access, promote increased adherence with a national practice guideline for pharmacists that benchmarks best practice in the area, and therefore increase the supply of, and enhance access to, the ECP by women.

\section{ETHICS AND DISSEMINATION}

Participants will be assured of their anonymity and that the primary purpose of the research is to identify the 
barriers and facilitators to accessing the ECP from community pharmacies in Australia.

Written informed consent will be taken from all pharmacists and women interviewed. All study participants will be provided with an explanatory statement containing information about the study, what participation involves and the contact details of the Monash University Ethics Committee, so that they are able to report any concerns or complaints about the study. All respondents have the right to refuse to answer any question posed by the interviewer, and can withdraw from the study prior to having approved the interview transcript.

The risk of physical or psychological harms from participation in the study will be negligible. The study only involves interviews with participants; however, given the potentially sensitive nature of the topic, the questions in both have been designed to be as objective as possible and have been worded carefully. Participants will not be identified by name in any report or publication resulting from the study data. There are no risks for the researchers.

The results from the study will be disseminated to all researchers and organisational partners associated with this study, who will brainstorm ideas for interventions that would address barriers and facilitators to access identified from the interviews. The study participants will receive an executive summary of the research findings. In addition, the findings will be written up for publication in peer-reviewed journals in specialist, general, national or international journals.

\section{Author affiliations}

${ }^{1}$ Centre for Medicine Use and Safety, Monash University, Melbourne, Victoria, Australia

2Judith Lumley Centre, La Trobe University, Melbourne, Victoria, Australia

${ }^{3}$ Department of General Practice, Monash University, Melbourne, Victoria, Australia

${ }^{4}$ Sydney Medical School, University of Sydney, Sydney, New South Wales, Australia

${ }^{5}$ School of Medicine and Pharmacology, University of Western Australia, Perth, Western Australia, Australia

${ }^{6}$ Deakin University and Flinders University, Melbourne, Victoria, Australia

${ }^{7}$ School of Pharmacy, University of Reading, Reading, UK

Twitter Follow Safeera Hussainy at @SafeeraHussainy

Acknowledgements Partners for the study are (in alphabetical order): API/ Priceline Pharmacy Group, Bayer Australia, La Trobe University, Marie Stopes International, Pharmaceutical Society of Australia, Quality Pharmacy Group, Women's Health Victoria, University of Sydney and University of Western Australia.

Contributors SYH is the chief investigator whose role is to supervise the research assistant, communicate with other investigators and study partners, establish partners, draft papers and disseminate findings as well as seek funding. AG is the research assistant and is employed for 2 days/week to manage the study by organising team meetings, preparing the ethics application, conducting all one-on-one participant interviews, drafting papers, collating findings and analysing data from the interviews. All other authors are investigators who have contributed to the study design. SYH and AG drafted the first version of this manuscript and all other authors revised it. All authors approved the submitted version
Funding This work is funded by the Faculty of Pharmacy and Pharmaceutical Sciences, Monash University Seed Grant Scheme, and Marie Stopes International (nil grant numbers assigned).

Competing interests None declared.

Patient consent Obtained.

Ethics approval Monash Human Research Ethics Committee (CF14/35512014001868).

Provenance and peer review Not commissioned; externally peer reviewed.

Open Access This is an Open Access article distributed in accordance with the Creative Commons Attribution Non Commercial (CC BY-NC 4.0) license, which permits others to distribute, remix, adapt, build upon this work noncommercially, and license their derivative works on different terms, provided the original work is properly cited and the use is non-commercial. See: http:// creativecommons.org/licenses/by-nc/4.0/

\section{REFERENCES}

1. Marie Stopes International. Real choices: women, contraception and unplanned pregnancy. Melbourne: Marie Stopes International, 2008:1-10.

2. Newman $\mathrm{P}$, Morrell S, Black M, et al. Reproductive and sexual health in New South Wales and Australia: differentials, trends and assessment of data sources. Ashfield, New South Wales: Family Planning NSW, 2011:p.xiii.

3. Raymond EG, Trussell J, Polis CB. Population effect of increased access to emergency contraceptive pills: a systematic review. Obstet Gynecol 2007;109:181-8.

4. Glasier A, Fairhurst K, Wyke S, et al. Advanced provision of emergency contraception does not reduce abortion rates. Contraception 2004;69:361-6.

5. Rodriguez MI, Curtis KM, Gaffield ML, et al. Advance supply of emergency contraception: a systematic review. Contraception 2013;87:590-601.

6. Polis $\mathrm{CB}$, Schaffer K, Blanchard K, et al. Advance provision of emergency contraception for pregnancy prevention (full review). Cochrane Database Syst Rev 2007;(2):CD005497.

7. Shaaban OM, Hassen SG, Nour SA, et al. Emergency contraceptive pills as a backup for lactational amenorrhea method (LAM) of contraception: a randomized controlled trial. Contraception 2013;87:363-9.

8. Raine TR, Harper CC, Rocca CH, et al. Direct access to emergency contraception through pharmacies and effect on unintended pregnancy and STIs: a randomized controlled trial. JAMA 2005;293:54-62.

9. Hobbs MK, Taft AJ, Amir LH, et al. Pharmacy access to the emergency contraceptive pill: a national survey of a random sample of Australian women. Contraception 2011;83:151-8.

10. Broekhuizen FF. Emergency contraception, efficacy and public health impact. Curr Opin Obstet Gynecol 2009;21:309-12.

11. Pharmaceutical Society of Australia. Supply of levonorgestrel as a Pharmacist Only medicine for emergency contraception (EC). Pharmaceutical Society of Australia Ltd, 2003.

12. Pharmaceutical Society of Australia. Supply of levonorgestrel as a Pharmacist Only medicine for emergency contraception (EC). Pharmaceutical Society of Australia Ltd, 2006.

13. Pharmaceutical Society of Australia. Guidance for provision of a Pharmacist Only medicine: levonorgestrel. Pharmaceutical Society of Australia Ltd, 2011

14. Hussainy SY, Stewart K, Pham M. A mystery caller evaluation of emergency contraception supply practices in community pharmacies in Victoria, Australia. Aust J Prim Health 2015;21:310-16.

15. Hussainy SY, Stewart K, Chapman CB, et al. Provision of the emergency contraceptive pill without prescription: attitudes and practices of pharmacists in Australia. Contraception 2011;83:159-66.

16. Mazza D, Harrison C, Taft A, et al. Unplanned pregnancy and emergency contraception in Australia: unsolved dilemmas. Aust N Z J Public Health 2014;38:110-11.

17. Hobbs M, Taft AJ, Amir LH. The emergency contraceptive pill rescheduled: a focus group study of women's knowledge, attitudes and experiences. J Fam Plann Reprod Health Care 2009;35:87-91.

18. Novikova N, Weisberg E, Fraser IS. Does readily available emergency contraception increase women's awareness and use? Eur J Contracept Reprod Health Care 2009;14:39-45.

19. Whelan A, Langille D, Hurst E. Nova Scotia pharmacists' knowledge of, experiences with and perception of factors interfering with their ability to provide emergency contraceptive pill consultations. Int $J$ Pharm Pract 2013;21:314-21. 
20. Downing SG, Payze C, Doyle-Adams S, et al. Emergency contraception over-the-counter: practices and attitudes of pharmacists and pharmacy assistants in far North Queensland. Aust N Z J Obstet Gynaecol 2011:51:527-31.

21. Dawson A, Tran N, Westley E, et al. Workforce interventions to improve access to emergency contraception pills: a systematic review of current evidence in low- and middle-income countries and recommendations for improving performance. BMC Health Serv Res 2015;15:180.

22. Francis JJ, O'Connor D, Curran J. Theories of behaviour change synthesised into a set of theoretical groupings: introducing a thematic series on the theoretical domains framework. Implement Sci 2012;7:35.

23. Cane J, O'Connor D, Michie S. Validation of the theoretical domains framework for use in behaviour change and implementation research. Implement Sci 2012;7:37.
24. Huijg J, Gebhardt W, Crone M, et al. Discriminant content validity of a theoretical domains framework questionnaire for use in implementation research. Implement Sci 2014;9:11.

25. Huijg J, Gebhardt W, Dusseldorp E, et al. Measuring determinants of implementation behavior: psychometric properties of a questionnaire based on the theoretical domains framework. Implement Sci 2014;9:33.

26. French S, Green S, O'Connor DA, et al. Developing theory-informed behaviour change interventions to implement evidence into practice: a systematic approach using the Theoretical Domains Framework. Implement Sci 2012;7:38.

27. Alexander KE, Brijnath B, Mazza D. Barriers and enablers to delivery of the Healthy Kids Check: an analysis informed by the Theoretical Domains Framework and COM-B model. Implement Sci 2014;9:60. 